\title{
MODELOS DE GESTIÓN DEL CONOCIMIENTO QUE INTEGREN TECNOLOGÍAS E-LEARNING EN LA EDUCACIÓN SUPERIOR
}

\section{MODELS OF KNOWLEDGE MANAGEMENT THAT INTE- GRATE E-LEARNING TECHNOLOGIES INTO HIGHER EDUCATION}

\begin{abstract}
University of the Amazonas has people who have an individual tacit knowledge in the area of computers programming, which play the role of a teacher. Teachers socialize this knowledge with their students through a masterful job in the classroom, complementing with work in groups and exhibitions, generating this way a public knowledge or which is also known as a collective tacit knowledge.

However, as it has been proved through an initial study, both teachers and students in the area of programming do not have the culture to be able to show this knowledge, for example, through the documentation of their classroom experiences. This causes the following issues, among others: from teachers' point of view: (i) it does not exist a socialization of the experiences which leads to make the same errors in teaching processes on each semester; (ii) the tacit knowledge of teachers are difficult to formalize, communicate and transfer. From the point of view of students: (i) it can be observed a high percentages of loss of the basic programming course; (ii) there are no other available educational media different from the Masterful class given by the teacher, which generates lack of motivation in the study.
\end{abstract}

One of the strategies that can help to solve these problems is what is known as a knowledge management model. The integration of e-learning tools into the knowledge management facilitates the processes of capture, organization, storage and transfer of the information, for later access and management of it. This article is a review of the state of the art about knowledge management models in education.

Key words: computer programming, educational media, e-Learning, higher education, knowledge management.

\section{RESUMEN}

La Universidad de la Amazonia cuenta con personas que tienen un conocimiento tácito individual en el área de la programación de computadores, los cuales desempeñan el rol de docente. Los docentes socializan este conocimiento a sus estudiantes mediante un
Ruth Escobar Sarria

Especialista en Teleinformática Docente de la Universidad de la Amazonia

ruthdaryescobar@gmail.com

Bogotá, Colombia

Carlos Enrique Montenegro Marín Doctor en Sistemas y Servicios Informáticos para Internet

Docente planta de la Universidad Distrital Francisco José de Caldas cemontenegrom@udistrital.edu.co Bogotá, Colombia

\section{Wilson Joven Sarria}

Especialista en docencia Universitaria y multimedia

Docente de la Escuela Colombiana de Carreras Industriales (ECCI)

wjoven@gmail.com

Bogotá, Colombia

José Fernando López Quintero Magister en Ingeniería de Sistemas Vicerrector de investigaciones de la Escuela Colombiana de Carreras Industriales (ECCI)

lopez.jose@ecci.edu.co

Bogotá, Colombia

Tipo: Artículo reporte de caso

Fecha de Recepción: Junio 10 de 2013 Fecha de Aceptación: Agosto 6 de 2013 
trabajo magistral en el aula de clase, complementado con trabajos en grupo y exposiciones, generando de esta forma un conocimiento público o lo que también se conoce como conocimiento tácito colectivo.

Sin embargo, como se ha evidenciado mediante un estudio inicial, tanto los docentes como los estudiantes del área de programación no tienen la cultura de exteriorizar este conocimiento, por ejemplo, mediante la documentación de sus experiencias de clase. Esto ocasiona, entre otros los siguientes problemas: desde el punto de vista de los docentes: (i) no existe una socialización de las experiencias lo que ocasiona que cada semestre se cometan los mismos errores en los procesos de enseñanza; (ii) los conocimientos tácitos de los docentes son difíciles de formalizar, comunicar y transferir. Desde el punto de vista de los estudiantes: (i) se denotan altos porcentajes de pérdida de la asignatura de programación básica; (ii) no se tiene a disposición medios educativos diferentes a la clase magistral impartida por el docente, lo que genera falta de motivación en el estudio.

Una de las estrategias que puede ayudar a solventar estos problemas es lo que se conoce como un modelo de gestión del conocimiento. La integración de herramientas e-learning en la gestión del conocimiento facilita los procesos de captura, organización, almacenamiento y transferencia de información, para posteriormente acceder a ella y gestionarla.

Este artículo es de revisión del estado del arte de la temática Modelos de gestión del conocimiento en la educación.

Palabras claves: educación superior, e-Learning, gestión del conocimiento, medios educativos, programación de computadores.

\section{INTRODUCCIÓN}

Aproximadamente el $60 \%$ de los estudiantes que ingresan al primer semestre de la carrera Ingeniería de Sistemas de la Universidad de la Amazonia, se encuentran con serias dificultades en el aprendizaje de los temas de programación básica de computadores, como por ejemplo expresar la solución de un problema real mediante un algoritmo, establecer el concepto de variable, poca interpretación del enunciado de un problema (el análisis), qué es lo que se quiere hacer y cómo es la solución lógica, donde se requiere una solución matemática no saben cómo aplicar el concepto.

Una posible solución a las inquietudes planteadas anteriormente, surgiría a partir de un modelo de gestión del conocimiento, el cual facilitaría recopilar y compartir las experiencia de los docentes en la enseñanza de los temas trabajados en la programación de computadores y los resultados obtenidos con la aplicación de las experiencias acopiadas en la resolución de problemas del mundo real, al igual que esas experiencias de aprendizaje que los estudiantes puedan compartir y que han facilitado el perfeccionamiento de habilidades en el desa- 
rrollo de software. Este modelo apoyado en tecnologías e-learning, podría facilitar la estrategia para el aprendizaje en línea a nivel de la formación en educación superior, que concatenadas con las características de los usuarios, facilitaría el acceso a ese conocimiento.

De esta manera, el desarrollo de esta investigación, busca a partir del diseño de un modelo de gestión de conocimiento y la integración de las tecnologías e-learning, estructurar y desarrollar una propuesta para mejorar los aprendizajes y formación en la línea de programación básica de computadores.

\section{CONTEXTO}

\subsection{Gestión del conocimiento}

Para entender el tema de gestión del conocimiento se presenta la siguiente definición:

La gestión de conocimiento hace énfasis en facilitar y gestionar actividades relacionadas con el conocimiento tales como la creación, captura, transformación y uso. Su función es planificar, implementar, operar y gestionar todas las actividades relacionadas con el conocimiento y los programas requeridos para la gestión efectiva del capital intelectual [49]

Los autores [7, 33, 23] sustentan las diferentes etapas de las que se compone el proceso de gestión del conocimiento. Estas inician con la localización de los conocimientos internos y externos disponibles; luego se identifica entre las fuentes de conocimiento, los conocimientos explícitos que se almacenaran y pondrán a disposición de quien los necesite y los conocimientos tácitos serán representados en un mapa de localización para identificar a las personas que los poseen; posteriormente, en la etapa de creación de conocimiento se extraerán los conocimientos explícitos para combinarlos y generar nuevo conocimiento; finalmente, la fase más importante de todo el ciclo consiste en asimilar y utilizar el conocimiento creado y almacenado.

\subsection{Modelo de gestión del conocimiento}

Existen varios modelos de gestión del conocimiento, entre los que podemos destacar los siguientes:El modelo de generación de conocimiento mediante dos espirales de contenido epistemológico y ontológico [34]; se destaca de este modelo que identifica dos tipos de conocimiento, el tácito (subjetivo) y el explícito (objetivo), asumiendo cuatro formas de conversión: de tácito a tácito (socialización), donde grupos de personas interactúan y comparten modelos mentales y experiencias; de tácito a explícito (exteriorización) referente a la discusión ó reflexión del conocimiento tácito para la creación de conocimiento conceptual a partir de metáforas y/o analogías; de explícito a explícito (combinación) que se genera a través de la distribución del conocimiento recién creado por redes de la organización, originando con ello conocimiento sistemático; y de explícito a tácito (interiorización) generación de nuevo conocimiento individual. Este modelo busca apoyar la generación de conocimiento de los empleados de una organización y no está enfocado en las instituciones educativas y los aprendizajes, además no utiliza herramientas e-learning para su implementación.

El modelo Andersen [2],enfoca la gestión del conocimiento desde dos perspectivas: la individual, basada en hacer explícito el conocimiento, y la organizacional, que busca crear la infraestructura de soporte, implantar procesos, la cultura, la tecnología y los sistemas que permitan capturar, analizar, sintetizar, aplicar, valorar y distribuir el conocimiento. El modelo de Andersen presenta la debilidad de subordinar la gestión del conocimiento a la captación de clientes exclusivamente, ignorando los aprendizajes.

El Modelo de Wiig [50] elabora un modelo integral sobre la gestión del conocimiento que engloba el proceso de creación, codificación y aplicación del conocimiento a la resolución de problemas en la organización. Se destaca que busca hacer una colección de información a partir de los aportes de los empleados, los ex- 
pertos, los libros y bases de datos y se realiza una selección de estos aportes para determinar que conocimientos en realidad son valiosos y se deben almacenar y difundir en toda la organización a través de un repositorio de conocimiento, apoyándose en las tecnologías de la información; además caracteriza los diferentes niveles de internalización del conocimiento, categorizando el conocimiento de los distintos actores en los siguientes niveles: novato, principiante, competente, experto y maestro. Este modelo no especifica el proceso de transformación del conocimiento, no distingue entre la creación y la comunicación del conocimiento.

\subsection{Gestión del conocimiento en la educación}

En los últimos años la gestión del conocimiento ha tomado fuerza en el área educativa, las instituciones educativas han empezado a articularla en el proceso de enseñanza y aprendizaje. La filosofía de la enseñanza y el aprendizaje no es acerca de cómo el conocimiento se transfiere de los maestros a los estudiantes, sino cómo los estudiantes pueden diseñar y crear nuevo conocimiento a partir de los conocimientos que han sido plasmados a través de libros, artículos, revistas, medios electrónicos, etc.

La Gestión del Conocimiento [38] en la educación busca la integración de todos los recursos humanos, procesos académicos y avances tecnológicos, involucrados en el diseño, la captura y ejecución de la infraestructura intelectual de una institución educativa. El enfoque se apoya en la elaboración y la gestión académica para aprender manteniendo el equilibrio entre las diversas entidades en un medio ambiente académico.

\subsection{Modelos de gestión del conocimiento en la educación}

Diferentes autores han abordado el tema de los modelos de gestión del conocimiento en la educación [41]. De ellos podemos destacar el planteamiento. Éste autor propone un modelo de gestión del conocimiento en educación que se compone de ocho módulos que interactúan y se retroalimentan del módulo central. Este módulo central denominado gestión del conocimiento utiliza competencias humanas, experiencias, conocimientos, habilidades, talentos, pensamientos, ideas, compromisos, innovaciones, prácticas y las integra a la información y los recursos que la organización utiliza para alcanzar sus objetivos estratégicos. Los demás módulos permiten adquisición del conocimiento, evaluación, uso del conocimiento, difusión del conocimiento, almacenamiento, aprendizaje, liderazgo utilizando tecnologías de la información, fortaleciendo los procesos de aprendizajes en la organización educativa, inculcando en las personas capacidades de crear y proporcionar el clima organizacional, de modo que la adquisición de conocimientos sea eficiente. Sin embargo, este modelo no define claramente las características de las TIC's utilizadas, lo que no permite identificar la utilización de herramientas e-learning en la implementación. Adicionalmente no es claro en el modelo si está dirigido a la educación superior, modalidad presencial y autónoma.

La demanda los modelos educativos [10] en la era de la postmodernidad son las nuevas formas de enseñar y de aprender, en contextos mixtos de aprendizaje (presencial+ virtual). El desarrollo de plataformas educativas, como parte de dichos contextos, requiere considerar modelos epistemológicos y de diseño tecnológico, que permitan obtener ambientes de trabajo pedagógico eficaces. Los escenarios de aprendizaje virtuales, pueden conformarse como comunidades inteligentes, capaces de pensarse a sí mismas y de gestionar su conocimiento, complementando la docencia presencial con docencia virtual. Se necesita asociar la integración curricular de tecnologías de la información y las comunicaciones con nociones de gestión del conocimiento, para agregar valor epistemológico al uso de tecnologías para el aprendizaje. También, para considerar y resolver la complejidad del diseño de estos ambientes, es necesario aplicar una metodología de desarrollo que sea incremental, interdisciplinaria y escalable, garantizando que los aspectos comunicacionales, pedagógicos, didácticos, tecnológicos y de gestión hayan sido debidamente considerados 
y resueltos equilibradamente. Estos autores en la propuesta presentan dos modelos de referencia, modelo pedagógico de gestión del conocimiento denominado circuito pedagógico de gestión del conocimiento y modelo incremental de prototipos denominado modelo incremental de prototipos para plataformas educativas. Además, a manera de ejemplos de aplicación de dichos modelos, tres experiencias de investigación y desarrollo, realizadas en distintos niveles del sistema educacional universitario, las que han tenido como finalidad conformar redes de contactos, para desarrollar redes de colaboración y afianzar redes de confianza, en torno a personas e instituciones que han compartido propósitos educativos de interés común. Estas experiencias son: red interuniversitaria para la investigación colaborativa, TIC-ETP: integración de tic en educación técnico profesional, específicamente este proyecto aplicó un modelo de gestión del conocimiento llamado modelo de gestión del conocimiento TIC-ETP y como tercera experiencia denominada formando profesores en redes de gestores de conocimiento de la misma manera se desarrolla un observatorio virtual para la gestión del conocimiento en educación. A pesar de ser claros estos aspectos en la propuesta, los autores no consideran de manera explícita las tecnologías e-learning.

La propuesta un modelo general utilizando las tecnologías de la información y las comunicaciones como medio para gestionar el conocimiento concebido durante el proceso de enseñanza y aprendizaje en una organización educacional denominado(GC + EA) [11]. Este modelo Parte de un contexto de aprendizaje, conformado por los docentes, los estudiantes, las estrategias de aprendizaje y el curriculum, luego identifica la infraestructura donde se promueve inicialmente el conocimiento, que puede suceder tanto en el aula tradicional, laboratorio, biblioteca, aula virtual, taller entre otros. Posteriormente involucra las tecnologías de la información y comunicación que son el soporte tecnológico que permite el acceso a fuentes de información y conocimiento. Por último se involucra a la comunidad académica virtual constituida por los docentes de la institución. La falencia que presenta este modelo es que el docente es quien dirige los aprendizajes y no el estudiante haciendo un trabajo autónomo, además no especifica el uso de tecnologías e-learning.

\subsection{Modelos que integran tecnologías e-learning}

En Colombia una herramienta e-learning de Gestión del conocimiento para el mejoramiento de la educación básica, secundaria y media vocacional [11]. Esta herramienta presenta una solución metodológica de gestión del conocimiento que integra tecnologías de la información y las comunicaciones y diferentes herramientas como www.destudio.com.co que permiten la interacción entre estudiantes y las comunidades estudiantiles. En esta propuesta se ilustran diferentes clases de e-learning, según su tipo (Autoformación y Colaborativos) y según su distribución (Stand-alone, en redes públicas y redes Internas). Para este desarrollo se ha definido una plataforma e-learning colaborativa, con contenidos de aprendizaje de calidad, además define una arquitectura donde solo se requiere que cada colegio tenga una conexión a internet y un portal. La idea de este esquema, aplicado a colegios, es que los estudiantes de distintos planteles interactúen entre sí aportando sus experiencias personales, sus vivencias y sus opiniones (conocimiento tácito) a una investigación para que estos generen nuevo conocimiento (conocimiento explícito). Los estudiantes pertenecientes a la investigación pueden ser de cualquier región del país.

Uno de los aspectos que se consideran desventaja en este tipo de soluciones es que no es evidente el trabajo del estudiante a nivel de educación superior, y no se apoyan en e-learning basado en agentes.

La propuesta presenta los estándares e-learning[1], siendo uno de los pilares fundamentales que ayudarán a gestionar con eficiencia uno de los activos más preciados de la denominada nueva economía: el conocimiento. Las instituciones, especialmente académicas, emprenden desarrollo de plataformas adaptadas a su imagen y semejanzas. Generalmente no 
cumplen ningún estándar e-learning en espacios educativos. Tradicionalmente la academia, conformada por universidades, institutos y colegios especializados, han aplicado las tecnologías e-learning de manera experimental y poco formal. Pocas instituciones la han aplicado de manera rigurosa. Con la aparición de internet, y específicamente e-learning, la entropía, la experimentación y la descentralización deben dar paso a una nueva forma de gestión, la cual deberá estar basada en las mejores prácticas, estándares y el cumplimiento de sanas referencias de gestión que contribuyan con la ansiada calidad educativa. La proliferación de contenidos, sistemas, plataformas y múltiples herramientas dispersas y sin ninguna posibilidad de operación entre ellas atenta contra los principios básicos de la productividad. Los estándares e-learning aplicados de manera sana y efectiva dentro de las instituciones educativas deben aproximarse hacia nuevas convergencias de eficiencia y calidad. Siendo este aspecto fundamental para gestionar conocimiento integrando e-learning. No obstante la importancia de la propuesta, el autor no considera los cambios tecnológicos que exigen un aprendizaje dinámico e inteligente apoyado en tecnologías e-learning basadas en agentes.

la importancia de la implantación de los estándares de e-learning [35]. Estándares que permitan a los usuarios incrementar la cantidad y calidad de contenidos compatibles entre plataformas. Sin embargo, los autores plantean que casi todos los estándares analizados están en fase de desarrollo. De igual manera han seleccionado para este estudio, entre la gran cantidad de organizaciones y grupos de trabajo, lo relativo a la definición de objetos de aprendizaje, procesos de aprendizaje y datos del alumno. Además, por la expansión del ámbito y aplicación del e-learning ha multiplicado la generación de los llamados objetos de aprendizaje, muchas veces sin controlar su calidad ni su verdadera utilidad. El objetivo de la creación de los objetos de aprendizaje es su utilización en cursos o, más genéricamente, en procesos de aprendizaje que se realizan en entornos tecnológicos distintos (interoperabilidad) y con objetivos y estructura diferentes pero con con- tenidos comunes (reusabilidad). Estos autores estudian la propuesta de arquitectura más extendida, sus elementos y relaciones, también expone la situación actual de estandarización de los elementos relacionados con los procesos formativos y los alumnos. Siendo estos aportes significativos en un modelo de gestión del conocimiento no se considera la tendencia de los aprendizajes en aprendizajes autónomos, dinámicos e inteligentes y avanzar en la estandarización de estos aspectos.

Un modelo para la creación de entornos de aprendizaje basados en técnicas de gestión del conocimiento [20]. Los autores definen un entorno de aprendizaje como el espacio donde hace posible gestionar el conocimiento. Se considera ventaja del modelo que se aplica a cualquier dominio de contenido intelectual, que permita actualizar los contenidos, que contenga estrategias genéricas de enseñanza que se adapten al comportamiento del estudiante y que fomente los diferentes tipos de aprendizaje. En el modelo que se presenta, se combinan la gestión del conocimiento con el uso de ontologías, áreas tradicionalmente no vinculadas en los entornos de aprendizaje. Para unificar los criterios sobre cuáles conceptos de conocimientos se presentarán, es necesario definir y formalizar los diferentes tipos de conocimiento a través de una ontología. Se incluye una conceptualización sobre los tipos de conocimiento, basada en ontologías reutilizables. El modelo fue implementado en Java. El entorno desarrollado denominado PLE:ASE (Programming Learning Environment: an Approach to Software for Education) fue aplicado y evaluado en un curso de primer año de programación orientada a objetos, con estudiantes de ingeniería en sistemas. Se constató que el uso del entorno permite al estudiante mejorar o ampliar las formas de resolución de problemas y sus capacidades para realizar la transferencia del conocimiento. Friss de Kereki presenta un modelo original, diferente a todos los analizados, y que es aplicable, pues su viabilidad quedó demostrada a través del sistema PLE:ASE, eficiente, de acuerdo a los resultados de la experimentación y basado en la gestión del conocimiento y sus técnicas, además permite explorar, evaluar y

Modelos de gestión del conocimiento que integren tecnologías e-learning en la educación superior 
manejar el conocimiento activamente. Sin embargo, la autora no considera el uso de estándares e-learning, que pasan a ser un apoyo a los aprendizajes en entornos tecnológicos, además en la propuesta no se plantea el uso de agentes inteligentes.

En su planteamiento parten de un sistema de e-learning como una solución prometedora a la demanda de un medio flexible de entrega de conocimientos para educar [52]. Proponen un marco conceptual y la arquitectura para el desarrollo de un sistema orientado a agentes de e-learning con el apoyo de la gestión del conocimiento para proporcionar un entorno flexible, el aprendizaje a su propio ritmo, y en colaboración con la menor restricción. El marco se basa en las tecnologías de los sistemas de e-learning, sistemas multi-agente (MAS), y KMSs. La arquitectura del sistema propuesto consta de tres niveles: a nivel de usuario, el nivel de dominio y, a nivel de web. El sistema proporcionará todos los servicios de apoyo básicos de enseñanza y aprendizaje relacionados, además de algunas características mayores que son proporcionados por los agentes dentro del sistema. El sistema también proporcionará los medios para capturar y compartir el conocimiento generado durante la utilización del sistema. Pero no se consideran un modelo de gestión del conocimiento donde el actor principal del proceso educativo sea el estudiante en su rol de aprendiz autónomo.

Identifican un modelo moderno de aprendizaje, que se compone de tres módulos de adquisición de conocimiento [28]. En primer lugar se ubican las fuentes de conocimiento con sus respectivos planes de estudio, luego se plantean los modelos de solución de problemas e identificación de competencias y termina con la adquisición de habilidades cognitivas a través de la práctica de conocimientos adquiridos. Se destaca del modelo que busca la transmisión de conocimiento tácito, como eje fundamental para actualizar conocimientos explícitos que se vuelven obsoletos en un corto tiempo y para ello se hace uso de las herramientas e-learning, enfocadas en la adquisición de conocimiento o resolución de problemas o una mezcla de los dos anteriores. Además los autores plantean en el modelo, el aprendizaje activo, en el cual estudiante es autónomo para decidir la forma de aprender determinada temática o buscar la solución a un problema de aprendizaje que se le presente; para hacer más dinámica la generación de conocimiento, el modelo se apoya de las herramientas tecnológicas y la inteligencia artificial, que permite que el alumno interactué con un robot que lo guía en su proceso de aprendizaje. No obstante el modelo no define una arquitectura y plataforma tecnológica a utilizar y se centra en la caracterización de varios software e-learning.

Un modelo denominado ConKMeL que consiste en una propuesta donde define un contexto de gestión del conocimiento integrando e-learning tradicional y e-learning inteligente [24]. La propuesta define una arquitectura de integración de e-learning, asi: se tiene un e-learning tradicional que consta de video y diapositivas, para apoyar la explicación de los contenidos, también referencias de documentos que a través de una capa de recursos de procesamiento, integran e-learning inteligente con una comunicación que especifica un contexto de búsquedas, un contexto de repositorio y un contexto de operación de agentes. También desde la capa de recursos de procesamiento se accede a un repositorio de recursos educativos, repositorio de experiencias y operación de agentes de experiencias con un nivel de control de acceso.

Este modelo también propone un enfoque de contexto semántico para apoyar e-learning inteligente, esto con el fin de descubrir y extraer el conocimiento por parte de los usuarios docentes y estudiantes, permitiendo búsquedas para facilitar los objetivos de conocimiento. Por otra parte este modelo permite un trabajo colaborativo en un ambiente de estudio, las operaciones de los instructores y los alumnos se basan en el seguimiento de cómo el conocimiento y experiencia podrían ser reutilizados en diferentes dimensiones semánticas. Sin embargo, todo acceso al conocimiento debe funcionar junto con un control basado en el contenido, esto para proteger la propiedad intelectual del conocimiento. No obstante el modelo no define 
los estándares y plataformas aplicados.

El papel de la gestión del conocimiento y elearning en el desarrollo profesional, ¿cómo la gestión del conocimiento y e-learning se pueden integrar y aprovechar para el aprendizaje y la educación?[42]. De la misma manera plantean que hay que tener en cuenta requisitos de aprendizaje, las principales características de los sistemas e-learning como la creación de cursos, estructura y secuencia de cursos basados en contenidos y los aspectos pedagógicos, psicológicos y educativos, así como los aspectos técnicos de la administración. Según estos autores la fase más importante de e-learning es la de aprendizaje, su contenido y su entorno. Identificando el aprendizaje autónomo e individual cuya comunicación puede ser sincrónica y asincrónica. También consideran los objetos de aprendizaje como la tecnología apropiada que se pueden trabajar en dispositivos móviles. Un aspecto importante es que los portales y repositorios se adaptan al tipo de aprendiz, sus características ajustan el curso a los estilos de aprendizaje y los objetos de aprendizaje, que son el fundamento para hablar de un aprendizaje colaborativo. A pesar de todas estas ventajas no se hace énfasis en los estándares y las plataformas.

El papel de la gestión del conocimiento seguirá siendo fundamental en la educación en la medida que las tecnologías avanzan y se integran para que la gestión del conocimiento y el elearning sean dinámicos y personalizados [18].

Se debe definir una clara estrategia de aprendizaje para hacer un desarrollo dinámico, flexible

\section{Referencias Bibliográficas}

[1] Uso de estándares e-learning en espacios educativos.. 2003. [En línea], consultado en Febrero 19 de 2012, disponible en: http://huespedes.cica.es/aliens/ revfuentes/campo_02.htm.

[2] A. Andersen; El Management en el Siglo XXI. Argentina: Ediciones Granica, S.A, y adaptable. La convergencia de la gestión del conocimiento y el e-learning, inicia en el momento que se provee conocimiento a través de los objetos de aprendizaje y se adaptan a la gestión de conocimiento. La desventaja está en definir la plataforma e-learning y los estándares.

\section{CONCLUSIONES}

Del estado del arte se constata que hay variada información sobre modelos pero, ninguno con las características de un modelo que integre la gestión del conocimiento, tecnologías elearning, aprendizajes de los temas de programación básica de computadores. Una carencia detectada es la falta de resultados concretos: se encontraron muchas propuestas pero pocas con información detallada de beneficios reales cuantificados.

Se plantea continuar con la generación de un modelo a partir de las falencias identificadas que integre un nivel de tecnologías. Definiendo plataformas como (Moodle y Sakai), arquitecturas donde se identifiquen repositorios como juegos, videos, páginas html, audio,ovas, archivos pdf,estándares y accesibilidad, interoperabilidad, repositorios, metadatos, para hacer de este modelo dinámico, interactivo, que de acuerdo a un perfil y nivel de estudiante, el pueda hacer de su aprendizaje independiente, autónomo y colaborativo en línea genere nuevo conocimiento.

De igual manera para trabajos futuros aplicar el modelo en otras áreas del conocimiento, en pro de mejorar los aprendizajes según las necesidades de los estudiantes.
[3] E. Angulo, M. Negrón; Modelo holístico para la gestión del conocimiento. Revista Científica Electrónica Ciencias Gerenciales, 11 (4), p.p 38-51, 2008.
P. Barker; Knowledge management for e-learning. Innovations in Education and 
Teaching International, 42 (2), p.p 111121, 2005.

[5] A. Barragán; Aproximación a una taxonomía de modelos de gestión del conocimiento. Intangible Capital, 5 (1), p.p 65-101, 2009.

[6] L. Bielski; E-learning's revised role, ABA Banking Journal, 97 (7), p.p 45-48, 2005

[7] A. Brooking; El capital intelectual: el principal activo de las empresas del tercer milenio, Editorial piado, Barcelona, 2007.

[8] Desarrollo de herramienta E-learning de gestión del conocimiento para el mejoramiento de la educación básica, secundaria y media vocacional en Colombia. [En Línea], consultado en Junio 20 de 2012, disponible en: http://bibliotecadigital.icesi.edu.co/ biblioteca_digital/bitstream/item/836/1/ DesarrolloherramientaElearning.pdf.

[9] Modelo de gestión del conocimiento, proceso de enseñanza y aprendizaje (GC + EA). [En Línea], consultado en Agosto 15 de 2012, disponible en: www.ie2010. $\mathrm{cl} /$ posters/IE2010-40.pdf.

[10] M. Careaga, A. Avendaño, M. Careaga;. Modelo gestión del conocimiento para plataformas de docencia universitaria mixta, (GC+TIC/DUM), nuevas ideas en informática educativa, p.p 355-376, 2009.

[11] Modelo pedagógico de gestión del conocimiento y modelo incremental de prototipos aplicados al desarrollo de plataformas educativas. [En línea], consultado en Enero 11 de 2013, disponible en: http://www.ie2010.cl/ posters/IE2010-75.pdf.

[12] Ch. Chantarasombat; Model a knowledge management for educational quality assurance in faculty of education, Mahasarakham University in Thailand. European Journal of Social Sciences, 11 (3), pp 428-440, 2007.

[13] Ch. Chantarasombat, R. Singapore; Developing a knowledge management model for educational quality assurance in faculty of education, Mahasarakham University, Educational Journal of Thailand, 2 (1), pp 10-22, 2008.

[14] Ch. Chantarasombat, B. Srisa-ard;
Developing a knowledge management model for self-reliant communities, Educational Journal of Thailand, 1 (1), 83-94, 2007.

[15] Y. Chou; The Implementation of Knowledge Management System in Taiwan's Higher Education. Journal of College Teaching \& Learning, 2 (9), p.p 35-42, 2005

[16] G. Correa, S. Rosero, H. Segura; Diseño de un modelo de gestión del conocimiento para la Escuela Interamericana de Bibliotecología. Revista interamericana de bibliotecología, 31 (1), pp 85-108, 2008.

[17] C. Cucu, M. Cristescu, C. Cristescu, C; Contributions to Using IT in education: an educational video player. Informatica Económica, 14 (2), p.p 108-119, 2010.

[18] D Lytras, M, A. Naeve, A Pouloudi; A knowledge management roadmap for e-learning: the way ahead, International Journal of Distance Education Technologies, 3 (2), pp 68-75, 2005.

[19] D Lytras, A. Naeve, A. Pouloudi; Knowledge management as a reference theory for e-learning: a conceptual and technological perspective, International Journal of Distance Education Technologies, 3 (2), PP1-12, 2005.

[20] D. Kereki; Modelo para la creación de entornos de aprendizaje basados en técnicas de gestión del conocimiento, Tesis de doctorado, Universidad Politécnica de Madrid. Madrid, 2003.

[21] A. Moral; Gestión del conocimiento. Editorial Thompson Editores, España, 2003

[22] Modelo de gestión del conocimiento (GC) aplicado a la universidad pública en el Perú. [En línea], consultado en Agosto 30 de 2012, disponible en: http://sisbib. unmsm.edu.pe/BibVirtual/Tesis/Basic/ Diaz MJ/Contenido.htm.

[23] F. Guadamillas; La gestión del conocimiento como recurso estratégico en un proceso de mejora continua, Alta Dirección, 217, pp199-209, 2001

[24] W. Huang, A. Mille; ConKMeL: a contextual knowledge management 
framework to support multimedia e-Learning. Multimed Tools Appl, pp 30, 205-219, 2007.

[25] Draft standard for learning technology - Learning Technology Systems Architecture (LTSA), IEEE. [En línea], disponible en: http://ltsc.ieee.org/wg1/ files/IEEE 148401 D09 LTSA.pdf.

[26] A. Ion; Knowledge management and eLearning. Informatica Economica, 4 (48), pp 15-28, 2008.

[27] G. Kende, E. Noszkay, G. Seres, G; Role of the knowledge management in modern higher education - the e-learning. AARMS, 6 (4), pp 559-573, 2007.

[28] B. Kogut, U. Zander; knowledge of the firm, combinative capabilities, and the replication of technology, Organization Science, 3, pp 383397, 1992.

[29] D. Lupa, S. Constantin, E. Doval; Developing and improving knowledge management processes in the education sector. Bulletin of the Transilvania University of Brasov, 2 (51), pp 87-90, 2009.

[30] N. Martínez; Gestión del conocimiento: aprendizaje individual versus aprendizaje organizativo. Intangible Capital, 2 (13), pp 308-326, 2006.

[31] Losprocesos decreación del conocimiento: el aprendizaje y la espiral de conversión del conocimiento. [En línea], consultado en Abril 12 de 2013, disponible en http://www.upct.es/ economia/ PUBLI-INO/LOS\%20PROCESOS $\% 20$ DE\%20CREACION\%20DEL\%20 C O N OC I M I E N T O - \% 20 E L \% 20 APRENDIZA.pdf.

[32] J. Medina; Elementos en la gestión del conocimiento. Una visión de teoría basada en recursos". XIV Congreso Nacional y $\mathrm{X}$ Congreso hispano-francés AEDEM, 2009.

[33] I. Nonaka, H. Takeuchi; The knowledgecreating company: How Japanese Companies Create the Dynamics of Innovation. New York-Oxford: Oxford University Press, 1993.

[34] Situación actual de estandarización de procesos de aprendizaje y su relación con la información sobre el alumno. [En línea], Consultado en Abril 1 de 2013, disponible en http://www.uoc.edu/ symposia/spdece05/pdf/ID04.pdf.

[35] R. Panckhurst, D. Marsh, D; Utilización de redes sociales para la práctica pedagógica en la enseñanza superior impartida en Francia: perspectivas del educador y del estudiante, en impacto de las redes sociales en la enseñanza y el aprendizaje, Revista de universidad y sociedad del conocimiento, 8 (1), pp 273-275, 2012.

[36] M. Pino; Aplicaciones de herramientas de e-learning a la docencia presencial, Formación e innovación educativa universitaria, 1 (4), pp 87-95, 2008.

[37] Application of knowledge management in management education: a conceptual framework. [En línea], consultado en Diciembre 4 de 2012, disponible en http://jatit.org/volumes/research-papers/ Vol3No3/3vol3no3.pdf.

[38] D. Rodríguez; Modelos para la creación y gestión del conocimiento: una aproximación teórica, Editorial Educar, pp 25-39, 2006.

[39] M. Rosenberg; E-learning: estrategias para transmitir conocimiento en la era digital, Editorial McGraw-Hill Bogotá, pp 70-73, 2001

[40] N. Salo; Knowledge Management in Education in Indonesia: An Overview. Global Journal of Human Social Science, 11 (1), pp 30-44, 2011.

[41] G. Sammour, The role of knowledge management and e-learning in professional developmen. knowledge and learning, 4 (5), pp 465-477, 2008

[42] Smith, S., y Foley S; Lessons in learning, e-learning, and training: perspectives and guidance for the enlightened, ProQuest Education Journals, 7 (2), pp 201-204, 2011

[43] Z. Su-zhen, Z. Hua; Framework of knowledge management in education based on knowledge grid, Journal of Communication and Computer, 6 (6), pp 60-66, 2009.

[44] M. Tippins, Implementing knowledge management in academia: Teaching the 
teachers. The International Journal of Educational Management, 17 (6/7), pp 339-345, 2003.

[45] UNESCO; Declaración mundial sobre la educación superior en el siglo XXI: visión y acción. [En línea], consultado en Junio 1 de 2012, disponible en http://www. unesco.org/education/educprog/wche/ declaration_spa.htm.

[46] G. Valerio, J. Valenzuela; Contactos de redes sociales en línea como repositorios de información, Revista de universidad y sociedad del conocimiento, 8 (1), pp 128141, 2011.

[47] J. Van;. A model of knowledge acquisition that refocuses knowledge management. Journal of Knowledge Management, 6 (1), 18-22, 2002.

[48] K.. Wiig; Management of knowledge: perspectives of a new opportunity, Arlington: The Wiig Group, France, 2012.

[49] K.. Wiig; Knowledge management foundations: thinking about thinking how people and organizations create, represent, and use knowledge, Volume 1 of knowledge management series, Arlington, TX: Schema Press, France, 1993.

[50] R. Wild, K. Griggs, T. Downing; A framework for e-learning as a tool for knowledge management. Industrial management + data systems, 102 (7), pp 371-380, 2002.

[51] H. Yau, E. Ngai, T. Cheng; Conceptual framework \& architecture for agentoriented knowledge management supported e-learning systems, Journal of distance education technologies, 3 (2), pp 48-67, 2005.

[52] J. Zahner; Teachers explore knowledge management and e-learning as models for professional development. techtrends, 46 (3), pp 11-16, 2002. 